\section{Pulmonary hypertension in ARDS: inflammation matters!}

\author{
Laura C Price, S John Wort
}

The recognition of pulmonary vascular complications in acute respiratory distress syndrome (ARDS) spans more than 40 years. Pulmonary hypertension $(\mathrm{PH})$ is defined as a mean pulmonary artery pressure of $\geq 25 \mathrm{~mm} \mathrm{Hg}$ at right heart catheterisation. $\mathrm{PH}$ is a recognised consequence of ARDS, with a high prevalence in early studies. The pathogenesis of $\mathrm{PH}$ in ARDS is likely to be multifactorial and disease stage-dependent. Further to the modifiable effects of positive pressure ventilation, ${ }^{1}$ potential underlying mechanisms of $\mathrm{PH}$ in ARDS include vessel obliteration, microthrombosis and pulmonary vasoconstriction due to hypoxia, hypercapnia and vasoactive mediator imbalance. Pulmonary vascular remodelling occurs later. ${ }^{2}$ Sepsis-related right-sided and also left-sided ventricular myocardial dysfunction may also contribute. Whatever the cause of $\mathrm{PH}$, the resulting right ventricular (RV) dysfunction is associated with increased morbidity and mortality, ${ }^{3} 4$ although it is not actually proven that RV failure is a mode of death in ARDS. Trials of pulmonary vasodilators, such as inhaled nitric oxide and prostacyclin, have met with disappointment in terms of outcomes, although there is fair criticism of the design of the studies and the heterogeneity of the populations involved. Notably, these studies targeted oxygenation rather than pulmonary haemodynamics; so, any potential contribution of improved RV function to better outcomes is untested. What is clear is that outcome has improved in ARDS with a parallel apparent reduction in prevalence of $\mathrm{PH}$ and RV dysfunction. Both may be due to changes in ventilation practice following the landmark protective ventilation studies, as limiting ventilator airway pressures directly reduces RV dysfunction through physiological mechanisms. ${ }^{5}$ However, even with the use of 'modern era' lower airway pressures, RV dysfunction remains in the region of $20 \%$ $25 \%{ }^{3}{ }^{4}$ and interestingly appears to be

Royal Brompton and Harefield NHS Foundation Trust, London, UK

Correspondence to Dr Laura Price, Royal Brompton and Harefield NHS Foundation Trust, Sydney Street, London SW3 6NP, UK; laura.price@rbht.nhs.uk associated with sepsis. ${ }^{3}$ So, what is the role of sepsis and, indeed, non-sepsis related inflammation to the pathogenesis of $\mathrm{PH}$ and RV dysfunction in the context of ARDS? We know that ARDS is, by its very nature, an inflammatory condition. It is associated with endothelial cell injury and dysfunction, ${ }^{6} 7$ events that may trigger acute pulmonary vasoconstriction, and also later remodelling processes. We also know that mechanical ventilation is bad for lungs, through processes such as ventilator-induced lung injury (VILI). High ventilatory pressures and flow increase lung cytokine levels, including interleukin (IL)-6 in $\operatorname{man}^{8}$ and also in relevant animal models of VILI, ${ }^{9}$ with levels falling as airway pressures are lowered. ${ }^{8} 9$ In addition, pulmonary arterial myography in rats ventilated with high tidal volumes demonstrates reduced $\alpha$-adrenergic induced vasoconstriction and reduced endothelium-dependent vasodilatation. ${ }^{10}$ It therefore seems likely that the increase in inflammation inherent to ARDS and also secondary to VILI drives the pulmonary vascular injury observed in this condition.

The article by Pandolfi and coworkers ${ }^{11}$ adds to the evidence that inflammatory mechanisms contribute to the pathogenesis of $\mathrm{PH}$ in ARDS. In particular, their study addresses whether IL- 6 and acid sphingomyelinase (aSMase) contribute to pulmonary vascular dysfunction in a rat model of inhaled lipopolysaccharide (LPS)-induced ARDS. They demonstrate that LPS induces both IL- 6 and ceramide production in rat pulmonary artery smooth muscle cells (PASMC) via an aSMase and transforming growth factor $\beta$-activated kinase- 1 dependent pathway, and that LPS increases serotonin-induced pulmonary vasoconstriction, also mediated by aSMase and IL-6. They show in vivo that pulmonary IL-6, IL-1 $\beta$ and endothelin (ET)-1 levels correlate with LPS-induced increases in pulmonary artery pressure, but interestingly that plasma levels of these cytokines, although also raised (but to a lesser degree), did not. aSMase inhibition using D609 prevented LPS-induced $\mathrm{PH}$ and reduced markers of inflammation in bronchoalveolar lavage, as well as IL-6 plasma levels. Specific IL-6 blockade was not used in vivo, but lung tissue IL-6 (and
IL-1 $\beta$ ) levels were suppressed by aSMase, suggesting their predominant production and target are in the lungs. Conversely, ET-1 levels were increased in both lung and plasma, but were not affected by aSMase.

While the effects of LPS, aSMase and IL-6 were to augment PH and vasoconstriction (in vitro and in vivo), the authors demonstrate in an isolated perfused rat pulmonary artery model that LPS-induced hypoxic pulmonary vasoconstriction (HPV) failure was also mediated by IL-6 and aSMase. In this model, LPS inhibited pulmonary vasoconstriction induced by hypoxia and phenylephrine, and acetylcholine-induced vasodilatation was blunted (although ET-1-induced contraction was unaffected). Signalling pathways were carefully dissected to show that LPS-induced HPV impairment via phenylephrine was mediated by inhibition of inducible nitric oxide synthase (iNOS), but not aSMase or IL-6, and that LPS-induced blunting of acetylcholineinduced relaxation was mediated via aSMase and iNOS inhibition, but not IL-6 blockade. The hyper-responsiveness of LPS to serotonin persisted despite iNOS inhibition. This suggests that HPV failure is complex and acts through IL-6 and aSMase-dependent mechanisms, and is not simply related to excess NO production by iNOS, which had been previously shown to be the case in a model of $\mathrm{PH}$ following intravenous microparticle injection in broiler chickens. ${ }^{12}$ In addition, the potent vasoconstrictor ET-1 and the LPS-induced hyper-responsiveness to serotonin must contribute to the overall increase in $\mathrm{PH}$ that, paradoxically, coexists with HPV failure. That aSMase inhibition using D609 could potentiate the vasoconstrictor response to hypoxia in these rat myography experiments was confirmed in human studies. The authors also nicely demonstrate in a rat model of partial airway occlusion that the LPS-induced delay in blood redistribution from unventilated alveoli was prevented by aSMase inhibition. The finding that the aSMase and IL-6 pathways contribute to HPV failure in this setting adds to the fascinating story of opposing vasoactive response between pulmonary and systemic vessels in relation to hypoxia, and to sepsis and endotoxinaemia, depending on the prominent vasoactive factor at play. Indeed, sphingolipids have been shown to mediate oxygen sensing and have opposing effects on vessels depending on the vascular tissue involved, ${ }^{13}$ with hypoxic vasoconstriction occurring in pulmonary arteries through inhibition of voltage-gated $\mathrm{K}(+)$ 
$(\mathrm{K}(\mathrm{v}))$ channels and activation of neutral SMase. $^{14}$

What do we already know about these mediators? There is evidence that sphingolipids are important in lung injury, sepsis, HPV and chronic lung diseases including COPD, idiopathic pulmonary fibrosis and pulmonary arterial hypertension $(\mathrm{PAH})$ as well as many types of cancer cells. For example, ceramide induces apoptosis in murine emphysema, ${ }^{15}$ susceptibility to bacterial infections in cystic fibrosis ${ }^{16}$ and IL-6 release from human lung fibroblasts. ${ }^{17} 18$ In human $\mathrm{PAH}$ and rodent hypoxic $\mathrm{PH}$, sphingosine kinase 1 is increased in the lungs and PASMC. SphK1 inhibition prevents hypoxic $\mathrm{PH}$, and overexpression promotes PASMC proliferation. ${ }^{19}$ Further to pro-proliferative effects, the proinflammatory role of sphingolipids may be relevant in $\mathrm{PAH}$, where dysregulated inflammation is recognised. The endotoxin-induced ceramide release from PASMC in this study ${ }^{11}$ is relevant to $\mathrm{PH}$ in ARDS and also chronic respiratory diseases, where acute exacerbations are characterised by a reversible increase in pulmonary arterial pressure, which is related to acute hypoxia (eg, in COPD). Chronic repeated inflammation is likely also to cause progressive pulmonary vascular remodelling and $\mathrm{PH}^{20}$ as suggested in patients with frequent COPD exacerbations. ${ }^{21}$

Hypoxia-inducible factor (HIF) regulation of cellular responses is likely to be relevant in this model of pulmonary vascular inflammation. Further to the response to cellular hypoxia, cytokines (including IL-6) and LPS can synergistically regulate HIF transcription. Through HIF-1 $\alpha$ binding, hypoxia activates nuclear factor (NF)- $\kappa \mathrm{B}$ expression, providing crosstalk between HIF and NF-кB pathways with resulting bidirectional signalling. $^{22}$ Downstream, HIF- $1 \alpha$ induces macrophage release of tumour necrosis factor- $\alpha$, IL-1 $\beta$ and vascular endothelial growth factor. ${ }^{22}$ This is likely to contribute to the acute pulmonary vasoactive effects described in this paper, as well as more chronic effects on pulmonary vascular cell proliferation with the onset of hypoxic vascular remodelling. ${ }^{23}$ In terms of relevance for sphingolipids, studies in hypoxic mouse myocardium suggest the conversion of ceramide from its precursor is hypoxia-related $;^{24}$ so, it is likely that HIF signalling is implicated in sphingolipid biology.

Overall, the study by Pandolfi et al supports the importance of inflammation and in particular IL-6 in endotoxin-induced endothelial dysfunction, failure of HPV and $\mathrm{PH}$. They provide a novel 'acute' pulmonary vascular action of IL-6, which adds to the well-studied chronic effects of IL-6 in hypoxia-driven $\mathrm{PH}$ and $\mathrm{PAH}$, as a key inflammatory and pro-proliferative mediator, as discussed by the authors. It may help to explain why protective ventilator strategies improve outcome, through lowering of IL-6 and other cytokines, mediated through an improvement in pulmonary vascular function, and suggests an era of targeting the vasculature in ARDS. Further studies of aSMase and IL-6 inhibition in VILI models would be of interest, as would unravelling how IL-6 acutely alters pulmonary vascular tone. Even more intriguing would be whether the inhibition of IL- 6 and aSMase in human ARDS would improve pulmonary vascular dysfunction (and outcome), and also the relevance of this axis to the acute worsening of $\mathrm{PH}$ in patients with acute exacerbations of chronic lung disease.

\section{Competing interests None declared.}

Provenance and peer review Commissioned; externally peer reviewed.

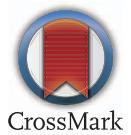

To cite Price LC, Wort SJ. Thorax 2017;72:396-397.

Published Online First 23 February 2017

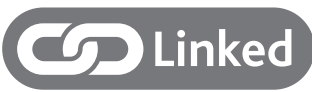

http://dx.doi.org/10.1136/thoraxjnl-2015-208067

Thorax 2017;72:396-397.

doi:10.1136/thoraxjnl-2016-209199

\section{REFERENCES}

1 Whittenberger JL, McGregor M, Berglund E, et al. Influence of state of inflation of the lung on pulmonary vascular resistance. J App/ Physiol 1960:15:878-82.

2 Snow RL, Davies P, Pontoppidan H, et al. Pulmonary vascular remodeling in adult respiratory distress syndrome. Am Rev Respir Dis 1982;126:887-92.

3 Boissier F, Katsahian S, Razazi K, et al. Prevalence and prognosis of cor pulmonale during protective ventilation for acute respiratory distress syndrome. Intensive Care Med 2013;39:1725-33.

4 Bull TM, Clark B, McFann K, et al. National Institutes of Health/National Heart, Lung, and Blood Institute ARDS Network. Pulmonary vascular dysfunction is associated with poor outcomes in patients with acute lung injury. Am J Respir Crit Care Med 2010;182:1123-8

5 Jardin F, Vieillard-Baron A. Is there a safe plateau pressure in ARDS? The right heart only knows. Intensive Care Med 2007:33:444-7.

6 Millar FR, Summers C, Griffiths MJ, et al. The pulmonary endothelium in acute respiratory distress syndrome: insights and therapeutic opportunities. Thorax 2016;71:462-73.

7 Tomashefski JF Jr, Davies P, Boggis C, et al. The pulmonary vascular lesions of the adult respiratory distress syndrome. Am J Pathol 1983;112:112-26.

8 Ranieri VM, Suter PM, Tortorella C, et al. Effect of mechanical ventilation on inflammatory mediators in patients with acute respiratory distress syndrome: a randomized controlled trial. JAMA 1999;282: 54-61.

9 Samary CS, Santos RS, Santos CL, et al. Biological impact of transpulmonary driving pressure in experimental acute respiratory distress syndrome. Anesthesiology 2015;123:423-33.

10 Menendez C, Martinez-Caro L, Moreno L, et al. Pulmonary vascular dysfunction induced by high tidal volume mechanical ventilation. Crit Care Med 2013;41:e149-55

11 Pandolfi R, Barreira B, Moreno E, et al. Role of acid sphingomyelinase and IL- 6 as mediators of endotoxin-induced pulmonary vascular dysfunction. Thorax 2017:72:460-71.

12 Wideman RF, Bowen OT, Erf GF, et al. Influence of aminoguanidine, an inhibitor of inducible nitric oxide synthase, on the pulmonary hypertensive response to microparticle injections in broilers. Poult $\mathrm{SC}$ 2006;85:511-27.

13 Moreno L, Moral-Sanz J, Morales-Cano D, et al. Ceramide mediates acute oxygen sensing in vascular tissues. Antioxid Redox Signal 2014;20:1-14.

14 Cogolludo A, Moreno L, Frazziano G, et al. Activation of neutral sphingomyelinase is involved in acute hypoxic pulmonary vasoconstriction. Cardiovasc Res 2009;82:296-302.

15 Medler TR, Petrusca DN, Lee PJ, et al. Apoptotic sphingolipid signaling by ceramides in lung endothelial cells. Am J Respir Cell Mol Biol 2008;38:639-46.

16 Brodlie M, McKean MC, Johnson GE, et al. Ceramide is increased in the lower airway epithelium of people with advanced cystic fibrosis lung disease. Am I Respir Crit Care Med 2010;182:369-75.

17 Laulederkind SJ, Bielawska A, Raghow R, et al. Ceramide induces interleukin 6 gene expression in human fibroblasts. J Exp Med 1995:182:599-604

18 Scarpa MC, Baraldo S, Marian E, et al. Ceramide expression and cell homeostasis in chronic obstructive pulmonary disease. Respiration 2013;85:342-9.

19 Chen J, Tang H, Sysol JR, et al. The sphingosine kinase $1 /$ sphingosine-1-phosphate pathway in pulmonary arterial hypertension. Am J Respir Crit Care Med 2014;190:1032-43.

20 George PM, Badiger R, Shao D, et al. Viral Toll Like Receptor activation of pulmonary vascular smooth muscle cells results in endothelin-1 generation; relevance to pathogenesis of pulmonary arterial hypertension. Biochem Biophys Res Commun 2012;426:486-91.

21 Wells JM, Washko GR, Han MK, et al. Pulmonary arterial enlargement and acute exacerbations of COPD. N Engl J Med 2012;367:913-21.

22 Whyte MK, Walmsley SR. The regulation of pulmonary inflammation by the hypoxia-inducible factor-hydroxylase oxygen-sensing pathway. Ann Am Thorac Soc 2014;11(Suppl 5):S271-6.

23 Cowburn AS, Crosby A, Macias D, et al. HIF2alpha-arginase axis is essential for the development of pulmonary hypertension. Proc Natl Acad Sci USA 2016:113:8801-6.

24 Azzam R, Hariri F, El-Hachem N, et al. Regulation of de novo ceramide synthesis: the role of dihydroceramide desaturase and transcriptional factors NFATC and Hand 2 in the hypoxic mouse heart. DNA Cell Biol 2013;32:310-19. 